\title{
Characteristics of Coping Strategies for Dysesthesia in Preoperative Patients with Compressive Cervical Myelopathy
}

\author{
Daisuke Higuchi \\ Department of Physical Therapy, Takasaki University of Health and Welfare, Takasaki, Japan
}

\begin{abstract}
Study Design: A cross-sectional study.
Purpose: This study aimed to clarify the characteristics of coping strategies for dysesthesia in preoperative patients with compressive cervical myelopathy.

Overview of Literature: Cognitive behavioral therapy is effective for patients with chronic pain in terms of modifying their negative behavior. To effectively perform cognitive behavioral therapy, it is necessary to assess coping strategies because of their important roles in health-related quality of life.

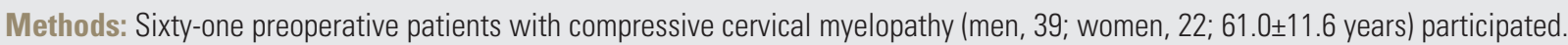
Coping strategies for dysesthesia (coping strategies questionnaire) and subjective symptoms (numerical rating scale for dysesthesia intensity and Japanese Orthopaedic Association cervical myelopathy evaluation questionnaire) were investigated.

Results: There were moderately significant correlations among the subcategory scores of the coping strategies questionnaire (Spearman's rank correlation coefficient $[r s] \leq 0.69, p<0.05)$; the praying/hoping and catastrophizing scores were significantly correlated with the numerical rating scale score of dysesthesia (both; $r s=0.34, p<0.01$ ); there were no correlations between the coping strategy scores and the cervical spine function and upper and lower extremity motor function scores of the Japanese Orthopaedic Association cervical myelopathy evaluation questionnaire; and there were no significant associations between the coping strategy scores and age, sex, and symptom duration.

Conclusions: Various combinations of coping strategies for dysesthesia were selected in patients with compressive cervical myelopathy, and frequency of use of the coping strategies was not related to the perceived severity of cervical myelopathy or demographic factors.
\end{abstract}

Keywords: Cervical vertebrae; Spinal cord disease; Paresthesia; Psychological adaptation

\section{Introduction}

Although compressive cervical myelopathy may give rise to various symptoms, many patients suffer abnormal sensations including stabbing pain and numbness (which are considered to be pain in the broad sense based on the definition of pain by the International Association for the Study of Pain [1]) below the neck [2]. Kamata and Satomi [3] reported that all preoperative patients with type I myelopathy based on Hattori's classification (i.e., the initial phase of compressive cervical myelopathy) complained of numbness, which is a type of dysesthesia. However, it

Received Sep 5, 2013; Revised Sep 29, 2013; Accepted Sep 29, 2013

Corresponding author: Daisuke Higuchi

Department of Rehabilitation, Harunaso Hospital, 5989 Nakamuroda, Tasakaki, Gunma 370-3347, Japan

Tel: +81-27-374-1135, Fax: +81-27-374-1135, E-mail: d_higuti@maroon.plala.or.jp 
is difficult to completely cure dysesthesia below the neck due to an imbalance between the spinothalamic and dorsal column systems (central nervous system dysfunction), which has been advocated as one of the mechanisms underlying dysesthesia [4].

In recent years, cognitive behavioral therapy (CBT) has been applied to patients with chronic pain to modify their resultant negative behavior. This therapy does not intend to remove the cause of pain, but to modify patients' beliefs and behaviors for the better management of their pain. Linton and Andersson [5] demonstrated the risk associated with long-term sick absence, and the use of physicians and physical therapy was significantly decreased by CBT in patients with spinal pain. Some researchers [68] succeeded in improving the pain intensity, pain-related disability, quality of life, and psychological status by using CBT in patients after lumbar surgery or spinal cord injury. Although CBT has an effect on neuropathic and/or mechanical pain caused by various spinal disorders, there are no studies on CBT for patients with compressive cervical myelopathy with dysesthesia.

It is necessary to assess coping strategies, defined as cognitive and behavioral efforts to manage external and internal stressors, to effectively perform CBT because coping strategies play important roles in health-related quality of life (HRQOL). For example, the coping strategy of catastrophizing was independently associated with health perception and disability regardless of the pain intensity [911]. However, neither the effect of CBT for patients with compressive cervical myelopathy with dysesthesia nor the characteristics of the coping strategies for dysesthesia in patients with cervical myelopathy are fully understood.

The present study aimed to clarify the characteristics of coping strategies for dysesthesia in preoperative patients with compressive cervical myelopathy before the CBT intervention trials. Relationships among coping strategies and relationships between coping strategies and other clinical measures, including the intensities of the dysesthesia, were investigated.

\section{Materials and Methods}

\section{Study design}

The present study was a cross-sectional study conducted from August 2011 to November 2012 in a general hospital with a spine care center in Japan. The present study was conducted after gaining approval from the hospital ethics committee.

\section{Participants}

A sufficient explanation of the present study was provided to preoperative patients with compressive cervical myelopathy, which was diagnosed based on the radiological findings. Patients who agreed in writing to participate in the present study were included. The following patients were excluded 1) those with motor and/or sensory dysfunction due to diabetes, circulatory disturbances of the peripheral vessels, osteoarthritis, cerebral vascular disease, and other spinal disorders affecting the thoracic or lumbar lesions and 2) those who could not sufficiently understand the questionnaire.

Surgery was planned for patients with progressive symptoms due to compressive cervical myelopathy, regardless of conservative treatment and/or difficulties in performing activities of daily living, including working, because of their symptoms. A posterior surgical approach was mainly applied in the present study. Open-door laminoplasty was conducted for patients with extensive anterior and/or posterior spinal cord compression. Wire and local bone grafting was used to fix open vertebral arches. The C2 and C7 muscles were conserved as much as possible. Laminectomy or posterior fusion using instrumentation was combined with laminoplasty if necessary. In some cases, anterior decompression and fusion for one or two intervertebral levels was conducted for patients with obvious anterior spinal cord compression, such as those with disc herniation. Discectomy and corpectomy were performed for decompression, and iliac or fibular grafting was then done.

\section{Assessments}

1) Coping strategies for dysesthesia

The Japanese short version of the coping strategies questionnaire (CSQ) [12], the internal consistency of which has been confirmed, was used. The CSQ contains six cognitive strategies (praying/hoping, catastrophizing, coping self-statements, diverting attention, reinterpretation, and ignoring) and two behavioral strategies (increasing pain behaviors and increasing activity levels). These eight strategies included two questions that were each graded on a 7-point Likert scale ( 0 , not at all; 6 , always); thus, the score of each strategy ranged from 0 to 12 points. A high- 
Table 1. Statistical overview of all assessed items ( $n=61)$

\begin{tabular}{|c|c|c|c|}
\hline Items & Range & Median (quartile deviation) & Maximum-minimum \\
\hline \multicolumn{4}{|l|}{ Coping strategy questionnaire } \\
\hline Praying/hoping & $0-12$ & $12(2.0)^{\mathrm{a})}$ & $0-12$ \\
\hline Catastrophizing & $0-12$ & $5(2.5)^{b)}$ & $0-12$ \\
\hline Coping self-statements & $0-12$ & $9(2.5)^{c)}$ & $0-12$ \\
\hline Diverting attention & $0-12$ & $7(3.0)^{d)}$ & $0-12$ \\
\hline Reinterpretation & $0-12$ & $5(2.0)^{b)}$ & $0-12$ \\
\hline Ignoring & $0-12$ & $4(2.5)^{b)}$ & $0-12$ \\
\hline Increasing pain behaviors & $0-12$ & $6(1.5)^{b)}$ & $0-12$ \\
\hline Increasing activity levels & $0-12$ & $6(2.5)^{d)}$ & $0-12$ \\
\hline Intensity of dysesthesia (NRS) & $0-10$ & $6(2.0)$ & $0-10$ \\
\hline \multicolumn{4}{|l|}{ JOACMEO } \\
\hline Cervical spine function & $0-100$ & $85(17.5)$ & $0-100$ \\
\hline Motor functions of upper extremity & $0-100$ & $89.5(10.5)$ & $36.8-100$ \\
\hline Motor functions of lower extremity & $0-100$ & $77.3(18.2)$ & $0-100$ \\
\hline Bladder function & $0-100$ & $87.5(9.4)$ & $43.8-100$ \\
\hline
\end{tabular}

NRS, numerical rating scale; JOACMEQ, Japanese Orthopaedic Association Cervical Myelopathy Evaluation Questionnaire.

Significant differences with all coping strategies with the exception of coping self-statements; ${ }^{\text {b) }}$ praying/hoping and self-statements; ${ }^{c /}$ catastrophizing, reinterpretation, ignoring, and increasing pain behaviors; and d)praying/hoping at $p<0.01$.

er score for each strategy indicated a higher frequency of use of the strategy.

\section{2) Subjective symptoms}

The 11-point numerical rating scale (NRS) was used (0, no dysesthesia; 10 , unbearable dysesthesia). Patients were asked about the average intensity of dysesthesia in the limbs that were most severely affected by dysesthesia during their daily living.

The Japanese Orthopaedic Association Cervical Myelopathy Evaluation Questionnaire (JOACMEQ) [13] was used to assess the subjective severity of the symptoms of compressive cervical myelopathy. The JOACMEQ classifies symptoms into the following five subcategories: cervical spine function, motor function of the upper extremities, motor function of the lower extremities, bladder function, and quality of life. Each subcategory ranges from 0 to 100 points ( 0 , patients felt severe dysfunction or poor health). Scores of all subcategories excluding quality of life were calculated in the present study.

\section{Analytical procedures}

First, the fundamental statistics of all assessed items were calculated, and the scores of the eight coping strategies of the CSQ were compared by the Steel-Dwass test. Second, Spearman's rank correlation coefficients among the CSQ coping strategy scores as well as between the CSQ coping strategy scores and scores of all assessed items, age, and symptom duration were calculated. In addition, the CSQ coping strategy scores were compared between men and women by the Mann-Whitney U test. All statistical analyses were performed using R version 2.8 (http://www. r-project.org/). Values of $p<0.05$ were considered statistically significant.

\section{Results}

A total of 61 preoperative patients with compressive cervical myelopathy (men, 39 [63.9\%]; women, 22 [36.1\%]; $61.0 \pm 11.6$ [standard deviation] years of age; symptom duration of $24.1 \pm 33.9$ months) participated in the present study. The numbers of patients with compressive cervical myelopathy caused by spondylosis, ossification of the posterior longitudinal ligament, and disc herniation were $44(72.1 \%), 15(24.6 \%)$, and $8(13.1 \%)$, respectively (including multiple conditions).

The fundamental statistics of all the assessed items and the results of the inter-coping strategy comparison are summarized in Table 1. Praying/hoping and self- 
Table 2. Spearman's rank correlation coefficients of CSO coping strategy scores ( $n=61$ )

\begin{tabular}{|c|c|c|c|c|c|c|c|}
\hline & $\mathrm{PH}$ & $\mathrm{CA}$ & $\mathrm{CS}$ & DA & $\mathrm{RE}$ & IG & IPB \\
\hline CA & $0.56^{\mathrm{a})}$ & $x$ & - & - & - & - & - \\
\hline CS & $0.59^{\mathrm{a})}$ & $0.44^{a)}$ & $x$ & - & - & - & - \\
\hline DA & $0.49^{\mathrm{a})}$ & $0.63^{\mathrm{a})}$ & $0.49^{a)}$ & $x$ & - & - & - \\
\hline RE & 0.23 & $0.36^{a)}$ & $0.46^{a)}$ & $0.46^{\mathrm{a}}$ & $x$ & - & - \\
\hline IG & 0.12 & 0.23 & $0.36^{a)}$ & $0.35^{\mathrm{a})}$ & $0.69^{a)}$ & $x$ & - \\
\hline IPB & $0.37^{\text {a) }}$ & $0.29^{b)}$ & 0.12 & $0.36^{a)}$ & 0.11 & 0.10 & $x$ \\
\hline $\mathrm{IAL}$ & $0.43^{\mathrm{a})}$ & $0.33^{\mathrm{a})}$ & $0.36^{a)}$ & $0.58^{\mathrm{a})}$ & $0.34^{a)}$ & $0.34^{\mathrm{a})}$ & $0.33^{\mathrm{a}}$ \\
\hline
\end{tabular}

CSQ, coping strategies questionnaire; $\mathrm{PH}$, praying/hoping; CA, catastrophizing; CS, coping self-statements; DA, diverting attention; RE, reinterpretation; IG, ignoring; IPB, increasing pain behaviors; IAL, increasing activity levels.

${ }^{\text {a) }} p<0.01$; ${ }^{\text {b) }} p<0.05$.

statements were significantly more frequently used than the other coping strategies, including catastrophizing $(p<0.01)$.

Spearman's rank correlation coefficients among the CSQ coping strategy scores ranged from 0.29 to 0.69 $(p<0.05)$ (Table 2). In addition, there were significant correlations between praying/hoping and catastrophizing and the NRS dysesthesia score (both, $\mathrm{rs}=0.34, p<0.01$ ); there were no significant correlations between the CSQ coping strategy scores and the JOACMEQ subcategory scores, with the exception of significant correlations between the catastrophizing and coping self-statement scores and the bladder function score ( $\mathrm{rs}=-0.26$ and $\mathrm{rs}=-$ $0.30 ; p=0.04$ and $p=0.02$, respectively). There were also no significant relations between the CSQ coping strategy scores and age, sex, and symptom duration.

\section{Discussion}

\section{Characteristics of participants}

Participants in the present study mainly comprised individuals in their fifties or sixties, and the number of men was greater than that of women. The age distribution and the men-to-women ratio in the present cohort agree with those of previous research [14]. In addition, the median NRS dysesthesia scores were 6 points out of a maximum score of 10 points, and the median scores of all four subcategory scores of the JOACMEQ were more than half of the maximum score (100 points). Therefore, many participants in the present study had moderate or slight compressive cervical myelopathy; few had severe dysesthesia or poor physical functioning.

\section{Selection of coping strategies for dysesthesia in preoperative patients with compressive cervical my- elopathy}

The praying/hoping and self-statement scores were significantly greater than the scores of the other coping strategies $(p<0.01)$. In the present study, preoperative patients with compressive cervical myelopathy tried to surmount the distress caused by the dysesthesia with prayer and/or by encouraging themselves rather than by diverting attention, reinterpreting, ignoring (i.e., an avoidance coping strategy), or catastrophizing. It is known that the avoidance coping strategies are a risk factor for chronicity of pain due to lumbar disc disease [15], and great fear associated with avoidance beliefs could predict low HRQOL in patients after lumbar disc surgery [16]. From the viewpoint of behavioral coping strategies, it is desirable to decrease pain behaviors and increase pain-free physical performance in patients with pain $[17,18]$. There was no significant difference between increased pain behavior and activity level scores in the present study. Moderate or slight dysesthesia caused by cervical myelopathy may not induce patients to first select avoidance coping, catastrophizing, and pain behavior strategies.

The diverting attention score was significantly correlated with the catastrophizing score $(r s=0.63, p<0.01)$, and the reinterpretation score was significantly correlated with the ignoring score $(\mathrm{rs}=0.69, p<0.01)$. Diverting attention is a strategy in which patients with compressive cervical myelopathy try not to recognize their own dysesthesia, while catastrophizing is a strategy in which patients try to confront their own dysesthesia. Although causality between diverting attention and catastrophizing was 
unclear, it was confirmed that even conflictive strategies could be simultaneously selected. Conversely, an association between reinterpretation and ignoring may be present based on the high similarity between these two strategies. In addition, Spearman's rank correlation coefficients among the CSQ coping strategy scores with the exception of those between the diverting attention and catastrophizing scores and the reinterpretation and ignoring scores were $<0.60$. This result reflects the fact that stereotypical combinations of coping strategies for dysesthesia are not always selected in patients with compressive cervical myelopathy.

The following factors are thought to affect the selection of coping strategies in patients with pain unrelated to cervical myelopathy: individual values, beliefs, psychological states, and social and environmental factors [19-21]. How patients with compressive cervical myelopathy cope with dysesthesia depends on many factors; however, these personal factors were not assessed in the present study. Therefore, relationships between the selection of coping strategies for dysesthesia and personal factors in patients with compressive cervical myelopathy are unclear. Age, sex, and symptom duration were not significantly related with the CSQ coping strategy scores, which is in agreement with previous research [22]. It was difficult to understand the tendency in the selection of coping strategies solely according to demographic factors. The abovementioned personal factors may be more important than demographic factors in the selection of coping strategies for dysesthesia in patients with compressive cervical myelopathy.

\section{Relationships between coping strategies and subjective symptoms}

The praying/hoping and catastrophizing scores were significantly correlated with the NRS dysesthesia score (both; $r s=0.34, p<0.01$ ). Patients with compressive cervical myelopathy who had strong dysesthesia were likely to select not only praying/hoping, but also catastrophizing. Women with chronic pain after a whiplash injury tended to select diverting attention, praying/hoping, catastrophizing, and increasing activity levels [23], and patients with chronic low back pain tended to select distraction and praying/hoping because their pain intensity was greater [11]. These results corresponded with the selected coping strategies for dysesthesia in the present study.
There were no significant correlations between coping strategy scores and three of the JOACMEQ subcategory scores (cervical spine function, motor function of the upper extremities, and motor function of the lower extremities). Therefore, whether the selection of coping strategies for dysesthesia depended on the level of motor function of the neck and upper and lower extremities or not could not be clarified. As stated above, individual values, beliefs, psychological states, and social and environmental factors [19-21] likely influence the selection of coping strategies to a greater degree than do motor functions.

\section{Study limitations and future prospects}

There are two major limitations in the present study. First, because the present study was a cross-sectional study, causalities among coping strategies for dysesthesia and between coping strategies and subjective symptoms in patients with compressive cervical myelopathy are unclear. Longitudinal data are needed to elucidate whether health care professionals can help patients with compressive cervical myelopathy to modify their coping strategies for dysesthesia in a beneficial way by CBT. Second, data regarding the social background and personality were not collected in the present study. These data are important to assess in order to understand the characteristics of coping strategies for dysesthesia in patients with compressive cervical myelopathy. Further research is needed to investigate the relationships between coping strategies and patients' social backgrounds and personalities in cases of compressive cervical myelopathy.

\section{Conclusions}

Various combinations of coping strategies for dysesthesia were selected in patients with compressive cervical myelopathy, and the frequency of use of these coping strategies was not related to the perceived severity of the cervical myelopathy or the demographic factors in the cohort. Therefore, assessment of the coping strategies for dysesthesia is recommended apart from the symptoms that are due to compressive cervical myelopathy before surgery. When we assess coping strategies for dysesthesia, we should particularly consider whether patients with cervical myelopathy with strong dysesthesia select the catastrophizing strategy. Patients who select the catastrophizing strategy for dysesthesia have a high risk of an ad- 
verse postoperative outcome. In the future, interventional studies are needed to clarify the influence of CBT on coping strategies for dysesthesia as well as the HRQOL.

\section{Conflict of Interest}

No potential conflict of interest relevant to this article was reported.

\section{Acknowledgments}

The author is grateful to the physical therapists at Harunaso Hospital for data collection and to the medical doctors at Gunma Spine Center for significant advice in the present study.

\section{References}

1. International Association for the Study of Pain. IASP Taxonomy [Internet]. Washington, D.C.: International Association for the Study of Pain; 2014 [cited 2013 Aug 29]. Available from: http://www.iasp-pain.org/ Content/NavigationMenu/GeneralResourceLinks/ PainDefinitions/default.htm.

2. Baron EM, Young WF. Cervical spondylotic myelopathy: a brief review of its pathophysiology, clinical course, and diagnosis. Neurosurgery 2007;60(1 Suppl 1):S35-41.

3. Kamata M, Satomi K. Classification of cervical myelopathy. MB Orthop 1997;10:1-6.

4. Beric A, Dimitrijevic MR, Lindblom U. Central dysesthesia syndrome in spinal cord injury patients. Pain 1988;34:109-16.

5. Linton SJ, Andersson T. Can chronic disability be prevented? A randomized trial of a cognitive-behavior intervention and two forms of information for patients with spinal pain. Spine (Phila Pa 1976) 2000;25: 2825-31.

6. Monticone M, Giovanazzi E. Usefulness of a cognitive behavioural and rehabilitative approach to enhance long lasting benefit after lumbar spinal stenosis and degenerative spondylolisthesis surgery: a case report. Eur J Phys Rehabil Med 2008;44:467-71.

7. Abbott AD, Tyni-Lenne R, Hedlund R. Early rehabilitation targeting cognition, behavior, and motor function after lumbar fusion: a randomized controlled trial. Spine (Phila Pa 1976) 2010;35:848-57.
8. Heutink M, Post MW, Bongers-Janssen HM, et al. The CONECSI trial: results of a randomized controlled trial of a multidisciplinary cognitive behavioral program for coping with chronic neuropathic pain after spinal cord injury. Pain 2012;153:120-8.

9. Santavirta N, Bjorvell H, Solovieva S, Alaranta H, Hurskainen K, Konttinen YT. Coping strategies, pain, and disability in patients with hemophilia and related disorders. Arthritis Rheum 2001;45:48-55.

10. Seres G, Kovacs Z, Kovacs A, et al. Different associations of health related quality of life with pain, psychological distress and coping strategies in patients with irritable bowel syndrome and inflammatory bowel disorder. J Clin Psychol Med Settings 2008;15: 287-95.

11. Woby SR, Watson PJ, Roach NK, Urmston M. Coping strategy use: does it predict adjustment to chronic back pain after controlling for catastrophic thinking and self-efficacy for pain control? J Rehabil Med 2005;37:100-7.

12. Ohtake K, Shimai S. Pain experience and coping strategies. Joseigaku-hyouron 2002;16:143-57.

13. Fukui M, Chiba K, Kawakami M, et al. JOA Back Pain Evaluation Questionnaire (JOABPEQ)/JOA Cervical Myelopathy Evaluation Questionnaire (JOACMEQ). The report on the development of revised versions. April 16, 2007. The Subcommittee of the Clinical Outcome Committee of the Japanese Orthopaedic Association on Low Back Pain and Cervical Myelopathy Evaluation. J Orthop Sci 2009;14:34865.

14. Lees F, Turner JW. Natural history and prognosis of cervical spondylosis. Br Med J 1963;2:1607-10.

15. Hasenbring M, Marienfeld G, Kuhlendahl D, Soyka D. Risk factors of chronicity in lumbar disc patients. A prospective investigation of biologic, psychologic, and social predictors of therapy outcome. Spine (Phila Pa 1976) 1994;19:2759-65.

16. Johansson AC, Linton SJ, Rosenblad A, Bergkvist L, Nilsson O. A prospective study of cognitive behavioural factors as predictors of pain, disability and quality of life one year after lumbar disc surgery. Disabil Rehabil 2010;32:521-9.

17. Van Oosterwijck J, Nijs J, Meeus M, et al. Pain neurophysiology education improves cognitions, pain thresholds, and movement performance in people with chronic whiplash: a pilot study. J Rehabil Res 
Dev 2011;48:43-58.

18. Epker J, Block AR. Presurgical psychological screening in back pain patients: a review. Clin J Pain 2001; 17:200-5.

19. Truchon M, Cote D, Schmouth ME, Leblond J, Fillion L, Dionne C. Validation of an adaptation of the stress process model for predicting low back pain related long-term disability outcomes: a cohort study. Spine (Phila Pa 1976) 2010;35:1307-15.

20. Hasenbring MI, Plaas H, Fischbein B, Willburger R. The relationship between activity and pain in patients 6 months after lumbar disc surgery: do pain-related coping modes act as moderator variables? Eur J Pain 2006;10:701-9.

21. Rodriguez Franco L, Cano Garcia FJ, Blanco Picabia A. Assessment of chronic pain coping strategies. Actas Esp Psiquiatr 2004;32:82-91.

22. Kraaimaat FW, Evers AW. Pain-coping strategies in chronic pain patients: psychometric characteristics of the pain-coping inventory (PCI). Int J Behav Med 2003;10:343-63.

23. Kivioja J, Jensen I, Lindgren U. Early coping strategies do not influence the prognosis after whiplash injuries. Injury 2005;36:935-40. 